\title{
Conductive heat transfer in a gas confined between two concentric spheres: From free-molecular to continuum flow regime
}

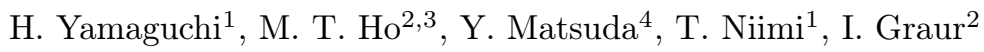 \\ ${ }^{1}$ Nagoya University, Department of Micro-Nano Systems Engineering, Furo-cho, Chikusa, \\ Nagoya, Aichi, 464-8603, Japan \\ ${ }^{2}$ Aix-Marseille Université, CNRS, IUSTI UMR 7343, 5 rue Enrico Fermi, 13453 Marseille, \\ France \\ ${ }^{3}$ University of Strathclyde, Department of Mechanical and Aerospace Engineering, James \\ Weir Fluids Laboratory, James Weir Building, 75 Montrose Street, Glasgow G1 1XJ, UK \\ ${ }^{4}$ Nagoya University, Institute of Materials and Systems for Sustainability, Furo-cho, \\ Chikusa, Nagoya, Aichi, 464-8603, Japan
}

\begin{abstract}
The conductive heat transfer through a gas confined between two concentric spherical shells maintained at different temperatures is investigated from the free-molecular to the continuum flow regime. The heat flux, measured using a recently proposed experimental system to extract the thermal accommodation coefficient, is compared with analytical expressions and numerical results. From this comparison it is found that in the free-molecular flow limit, the experimental data are well explained by the analytical expression for the arbitrary radius and temperature ratios of the spherical surfaces. In the continuum limit, the temperature dependence of the thermal conductivity coefficient should be considered in the analytical expression. In the transitional flow regime, a revised function for the heat flux interpolation is proposed to give better fitting to the numerical results. By employing these knowledge, the thermal accommodation coefficient extraction procedure for the system is revised, and it is shown that the re-calculated accommodation coefficient allows to reproduce well the measured heat flux.
\end{abstract}

Keywords: heat transfer; vacuum; Knudsen number; kinetic model; concentric spheres; thermal accommodation coefficient

\section{Introduction}

The heat transfer through a rarefied gas confined between two surfaces with different temperatures is a fundamental issue which has been studied for a long time $[1,2]$. In parallel to the study of the theoretical aspects of the heat transfer as its dependence on the gas nature, composition and pressure various practical 
applications were found, such as the principle of operation of the Pirani sensor, which uses the dependence of heat flux on the pressure for the pressure measurements. Despite the long history of investigations of heat transfer between the two surfaces, there are several aspects which remain not very well known until now. One of these problems lies in the interaction of gas molecules with surfaces, which significantly affects the intensity of the heat transfer between the surfaces. This interaction may be explained in terms of thermal accommodation coefficient. The thermal or energy accommodation coefficient $\alpha$ is defined as $[1,2]$,

$$
\alpha=\frac{\overline{E_{\mathrm{i}}}-\overline{E_{\mathrm{r}}}}{\overline{E_{\mathrm{i}}}-\overline{E_{\mathrm{s}}}}
$$

where $\bar{E}$ is the mean energy of the molecules colliding to a surface; the subscripts $\mathrm{i}$ and $\mathrm{r}$ correspond to the incident and reflected molecules, respectively, the subscript $\mathrm{s}$ corresponds to the molecules fully accommodated to the surface. This coefficient is useful in the analysis and the management of heat transfer in micro- and nano-devices, where gas flow should be treated as rarefied, even if the operating pressure is an atmospheric pressure, due to their small characteristic dimension. Additionally, the surface-to-volume ratio of the fluid for microand nano-devices becomes much larger than that for the conventional devices, therefore, the gas-surface interaction plays an essential role[3, 4, 5, 6].

In this study, we focused on the heat transfer problem between two concentric spheres. This geometry was employed in a novel measurement system of the thermal accommodation coefficient, which characterizes the mean energy transfer through the gas-surface interaction [7]. This measurement system is based on the low pressure method, firstly introduced by Knudsen, later employed by many researchers [1]. This method employs the particular property of heat transfer at low pressure: the heat transfer between two surfaces maintained at different temperatures is proportional to the pressure between them and the thermal accommodation coefficient. Therefore, the heat flux through rarefied gas confined in concentric spherical shells is measured as a function of pressure, then the thermal accommodation coefficient is extracted. The novel measurement system is able to measure the thermal accommodation coefficient on non-metal surfaces, which is rarely reported in the literature due to specificity of the measurement methods $[2,8]$, and it is especially important for micro- and nano-devices because of the materials employed.

Recently, the heat transfer between the two concentric spheres system is also simulated [9] on the basis of the nonlinear S-model kinetic equation [10]. In the free-molecular, slip and continuum flow regimes, the analytical expressions are provided for arbitrary temperature and spheres' radius ratios. In the transitional regime, the S-model kinetic equation is solved numerically. Then, the limits of the applicability of the obtained analytical expressions and the previous empirical relation are established by confronting the numerical and analytical solutions.

The objective of this study is to evaluate the accuracy of the empirical heat flux expression between the free-molecular and continuum flow regime, and re- 
vise the expression valid for the whole range of the flow regime. The analytical heat flux expressions in the free-molecular and continuum flow regimes and the numerical data in the transitional flow regime, obtained in Ref. [9], are used to derive this revised heat flux expression. Then, based on the measurements of the heat flux and the pressure between two spherical shells, provided in Ref. [7], the values of the thermal accommodation coefficients are derived using proposed revised expression of the heat flux. Finally, the numerical simulations are carried out using the S-model kinetic equation with the obtained thermal accommodation coefficients, and the measured and simulated heat fluxes are compared to validate the revised expression.

\section{Analysis of Experimental Heat Flux Data}

The experimental data on the heat flux reported in Ref. [7] are analyzed in the following. The two concentric spherical shells configuration is chosen as the experimental setup to measure the thermal accommodation coefficient on non-metal surfaces. A tiny heater, maintained at temperature $T_{H}$ by an analog electrical circuit, is fixed at the center of a spherical vacuum chamber. The tiny heater has a thin flat-plate shape, and the test sample surfaces are attached to the heater. The surface of the vacuum chamber made by Pyrex is maintained at the temperature $T_{C}$ by immersing the chamber in a water bath. The inner radius of the chamber is equal to $R_{C}=49.5 \mathrm{~mm}$. The heat flux from the tiny sample to the chamber surface is measured together with the pressure in the chamber. By using the expression relating the heat flux between the surfaces and the pressure, the thermal accommodation coefficient is derived. The configuration of the experimental setup is explained in detail in Ref. [7].

The heat flux from a heated surface surrounded by a monatomic gas in the the free-molecular flow regime is expressed as,

$$
q_{F M}=\frac{\alpha}{2} \frac{\bar{v}}{T} p\left(T_{H}-T_{C}\right), \quad \bar{v}=\sqrt{\frac{8 k T}{\pi m}},
$$

here $T, p$ and $\bar{v}$ are the temperature, pressure, and mean molecular speed of the gas, respectively; $k$ is the Boltzmann constant and $m$ is the molecular mass of the gas. It is important to underline that the heat flux is proportional to the pressure of the gas and, therefore, to the gas number density. The heat flux between two surfaces maintained at different temperatures in the free-molecular flow regime, Eq. (2), is independent of the geometrical configuration of the system, see Refs. $[8,3,9]$. The accommodation coefficient $\alpha$ can be obtained by fitting heat fluxes measured as a function of pressure by Eq. (2).

However, it is difficult in a simple apparatus to maintain the free-molecular flow regime (i.e., low pressure environment) owing to leakage. An alternative way is to build a high vacuum experimental setup; however, in this case, the measurement system becomes huge and costly. In addition, it is also not easy to measure very small heat flux at low pressure environment. To realize the measurement in a low-cost simple apparatus, a much higher pressure condition is 
favorable for the measurement. A more general model for the heat flux through a gas from a heated surface has to be implemented for the accommodation coefficient extraction for this condition extended to the higher pressure. For the whole flow regimes, i.e. from the free-molecular to continuum flow regimes, the expression of the heat flux $q$ can be approximated by a simple empirical interpolation of the free-molecular limit heat flux $q_{F M}$, Eq. (2), and the continuum limit heat flux, $q_{C}$, as it was done in Refs. $[8,3,11,12]$, and it is expressed as

$$
\frac{1}{q}=\frac{1}{q_{F M}}+\frac{1}{q_{C}} .
$$

For the continuum limit the heat flux $q_{C}$ is described by Fourier's law and it is independent of pressure and dependent upon the flow geometry. By making the size of the internal heated surface relatively small compared to the external surface of the vacuum chamber, the heat transfer problem is approximated by a simple spatially symmetric heat transfer between two concentric spherical shells, even though the shape of this internal heated surface is not a sphere but a flat plate, as mentioned above [7]. Following this model of two spherical shells, we can calculate the radius of the internal "virtual" sphere from the surface areas equality: the surface of the internal "virtual" sphere of a radius $R_{H}$ has the same surface area as the real heated surface. From this equality, the radius of "virtual" internal sphere is estimated as $R_{H}=4.95 \mathrm{~mm}$. Thus, the radius ratio of the concentric spherical shells, $\mathcal{R}=R_{C} / R_{H}$, is equal to 10 and it is relatively large. By assuming the concentric spherical shells geometry, the theoretical heat flux at the continuum limit $q_{C}$ in Eq. (3) is expressed as

$$
q_{C}=\kappa\left(T_{H}-T_{C}\right) \frac{R_{C} R_{H}}{R_{C}-R_{H}} \frac{1}{R_{H}^{2}},
$$

where $\kappa$ is the thermal conductivity of the gas. The temperature inside the spherical vacuum chamber is assumed to be equal to the temperature of the surface of the external spherical shell $T_{C}[7,8]$. Therefore, in this analysis, the temperature dependence of the thermal conductivity $\kappa$ along the radial direction is not taken into account, and the thermal conductivity at the temperature of the external spherical shell, $T_{C}$, is used for the entire region inside the vacuum chamber: $\kappa=\kappa\left(T_{C}\right)$. In addition, the pressure is assumed to be constant between the shells.

To minimize an error which can come from the use of the empirical relation, Eq.(3), the pressure condition is limited below $1.6 \mathrm{~Pa}$ in the experiment so that the measurement is realized in the near free-molecular regime.

In order to test this new developed experimental setup, first a platinum sample is used. A platinum foil with a thickness of $10 \mu m$ (Nilaco) is selected as a sample surface. Five values of the hot sphere surface temperature, $T_{H}$, are stated in the experiments, see Table 1 . The cold sphere temperature, $T_{C}$, is almost the same for all five cases and equal to a room temperature. A number is attributed for each hot temperature value to simplify the reference. The averaged accommodation coefficients, extracted by the described above procedure from three measurements of the heat flux and pressure for each case, are 


\begin{tabular}{|c|c|c|c|c|c|c|}
\hline \multicolumn{2}{|c|}{ cases } & 1 & 2 & 3 & 4 & 5 \\
\hline \multirow{3}{*}{$\mathrm{He}$} & $T_{H}(\mathrm{~K})$ & 335 & 364 & 395 & 424 & 453 \\
\hline & $T_{C}(\mathrm{~K})$ & 294 & 294 & 294 & 294 & 294 \\
\hline & $\alpha$ & 0.280 & 0.292 & 0.308 & 0.322 & 0.338 \\
\hline \multirow{3}{*}{$\mathrm{Ar}$} & $T_{H}(\mathrm{~K})$ & 335 & 364 & 394 & 424 & 453 \\
\hline & $T_{C}(\mathrm{~K})$ & 293 & 293 & 293 & 293 & 294 \\
\hline & $\alpha$ & 0.850 & 0.867 & 0.856 & 0.864 & 0.886 \\
\hline \multirow{3}{*}{$\mathrm{Xe}$} & $T_{H}(\mathrm{~K})$ & 335 & 364 & 394 & 424 & 453 \\
\hline & $T_{C}(\mathrm{~K})$ & 294 & 294 & 294 & 294 & 294 \\
\hline & $\alpha$ & 1.024 & 1.045 & 1.066 & 1.053 & 1.065 \\
\hline
\end{tabular}

Table 1: Experimental temperature conditions and mean energy accommodation coefficients based on measurements.

provided in Table 1. Surface temperatures were different for each measurement, and the mean surface temperatures are listed, with any variation from the mean value less than $0.2 \mathrm{~K}$. The relative standard errors of the accommodation coefficient did not exceed $1.6 \%$, showing good repeatability of the measurements. However, it was not so simple to estimate the measurement accuracy of the system [7], and the number of significant digits was decided from the size of the above mentioned relative standard error. From Table 1, the accommodation coefficients exceed unity in some cases. This could be coming from above mentioned several assumptions made in the extraction procedure. Therefore, more accurate expression to describe the heat flux is important to improve the existing methodology of the accommodation coefficient extraction.

\section{Analytical Solution and Numerical Simulation}

In this section, we present the detailed analytical and numerical analyses on the heat flux problem between two concentric spherical shells in all flow regimes following Ref. [9]. The assumptions used for derivation of all analytical expressions are provided. These analyses allow to estimate the error, which is made, when using the analytical expression for a set of physical parameters, and also it allows the improvement on the accuracy of the accommodation coefficient extraction procedure.

The heat transfer between two spherical shells, of the arbitrary radii $R_{H}$ and $R_{C}$, for the internal and external shells, respectively, is analyzed for various temperatures of the shells' surfaces $T_{H}$ and $T_{C}$. The detailed description of the developed approaches can be found in Ref. [9]. We provide here only one part of the results indispensable for the accommodation coefficient extraction procedure. To characterize the level of the gas rarefaction, it is convenient to introduce the rarefaction parameter as following

$$
\delta_{0}=\frac{R_{0}}{\ell}, \quad \ell=\frac{\mu_{0} v_{0}}{p_{0}}, \quad v_{0}=\sqrt{\frac{2 k T_{0}}{m}} .
$$

Here $R_{0}$ is the reference length of the problem, $\ell$ is the equivalent mean free path, $p_{0}$ is the reference pressure, $p_{0}=n_{a v} k T_{0}, n_{a v}$ is the number density 
averaged over the physical space $n_{a v}=3 /\left(R_{C}^{3}-R_{H}^{3}\right) \int n(r) r^{2} d r, r$ is the radial coordinate of physical region between the concentric spheres with the origin at their centers, $n(r)$ is the gas number density, which depends on $r$ coordinate only, $\mu_{0}$ and $v_{0}$ are the gas viscosity and the most probable molecular velocity at the reference temperature $T_{0}$, respectively. For convenience, the reference values in Eq. (5) are taken as follows

$$
T_{0}=T_{C}, \quad R_{0}=R_{C}-R_{H} .
$$

The definition of the rarefaction parameter allows us to choose the appropriate modeling as a function of the rarefaction parameter $\delta_{0}$ value. The cases of $\delta_{0}=0$ and $\delta_{0} \rightarrow \infty$ correspond to the free-molecular flow and continuum limits, respectively. It is to note that the gas rarefaction parameter $\delta_{0}$ in Eq. (5) is inversely proportional to the Knudsen number.

\subsection{Free-molecular flow regime}

In the free-molecular flow limit $\left(\delta_{0} \rightarrow 0\right)$, the Boltzmann equation (or model kinetic equations) [13] can be solved analytically for arbitrary temperature and radius ratio, because the collision term in its right-hand side can be neglected in this case. We assume here the complete accommodation of the molecules with the surface on the external sphere $\alpha_{C}=1$ following the discussion in the experimental analysis [8, 7], and on the internal sphere surface, the Maxwelltype diffuse-specular reflection with $\alpha_{H}=\alpha$ is assumed. The heat flux at any point between the spheres reads

$$
\begin{aligned}
q_{F M}(r) & =\frac{\alpha}{2} p_{0} \bar{v}(\mathcal{T}-1) K_{F M}\left(\frac{R_{H}}{r}\right)^{2} \\
K_{F M} & =\left[1+\frac{\alpha}{2}\left(\sqrt{\mathcal{T}^{-1}}-1\right)\left(1-\frac{(\mathcal{R}+1) \sqrt{\mathcal{R}^{2}-1}}{\mathcal{R}^{2}+\mathcal{R}+1}\right)\right]^{-1}
\end{aligned}
$$

where $\mathcal{T}$ is the temperature ratio, $\mathcal{T}=T_{H} / T_{C}$. It is interesting to note that the expression of the heat flux depends not only on the temperature ratio $\mathcal{T}$, but also on the radius ratio $\mathcal{R}$.

\subsection{Transitional regime}

Contrarily to the free-molecular flow regime, where the analytical solution of the kinetic equation was obtained, only numerical solution is possible in the transitional regime. Therefore, in this regime the S-model kinetic equation [10] was solved numerically for various range of the rarefaction parameter, shell's temperature and radius ratios. The details can be found in [9]. 


\subsection{Continuum regime}

In the continuum limit, the temperature variation between two concentric spheres may be obtained from the energy balance

$$
\frac{\partial}{\partial r}\left(r^{2} \kappa \frac{\partial T}{\partial r}\right)=0 .
$$

It is to note that here the hypothesis of zero macroscopic gas velocity is used and only the conduction heat transfer is considered. The Fourier law can be applied to calculate the heat flux

$$
q=-\kappa \frac{d T}{d r} .
$$

For the monatomic gases the gas thermal conductivity is related to the gas viscosity as follows

$$
\kappa=\frac{15}{4} \frac{k}{m} \mu .
$$

In order to define the dependence of the viscosity on the temperature, the molecular interaction potential must be specified, and we use the inverse power law potential [14] in the following. This model leads to a power law temperature dependence for the viscosity coefficient

$$
\mu=\mu_{0}\left(\frac{T}{T_{0}}\right)^{\omega},
$$

where $\omega$ is the viscosity index, which is equal to 0.5 for the Hard Sphere model and 1 for the Maxwell model. In the presented here analysis, the Variable Hard Sphere model (VHS) [14] is used, where the viscosity index varies with the gas nature; it is equal to 0.66, 0.81 and 0.85, for Helium, Argon and Xenon, respectively. Taking into account the relation between the thermal conductivity and viscosity, Eq. (10), the thermal conductivity has similar temperature dependence to the viscosity.

In the continuum limit, the gas temperature in the vicinity of a wall is equal to the wall temperature, so Eqs. (8) and (9) are solved analytically for the arbitrary temperature and radius ratios. The heat flux distribution takes form:

$$
\begin{aligned}
q_{C}(r) & =\kappa\left(T_{C}\right) K_{C}\left(T_{H}-T_{C}\right) \frac{R_{C} R_{H}}{R_{C}-R_{H}} \frac{1}{r^{2}}, \\
K_{C} & =\frac{\mathcal{T}^{\omega+1}-1}{(\omega+1)(\mathcal{T}-1)} .
\end{aligned}
$$

It is worth to note that contrarily to expression (4), the temperature dependence of the heat conductivity is taken into account in this expression. 


\begin{tabular}{|ll|lllll|}
\hline \multicolumn{2}{|c|}{ cases } & 1 & 2 & 3 & 4 & 5 \\
\hline $\mathrm{He}$ & $\alpha$ & $0.280( \pm 0.0 \%)$ & $0.292( \pm 0.0 \%)$ & $0.308( \pm 0.0 \%)$ & $0.322( \pm 0.0 \%)$ & $0.338( \pm 0.0 \%)$ \\
$\mathrm{Ar}$ & $\alpha$ & $0.849(-0.12 \%)$ & $0.867( \pm 0.0 \%)$ & $0.855(-0.12 \%)$ & $0.863(-0.12 \%)$ & $0.885(-0.11 \%)$ \\
$\mathrm{Xe}$ & $\alpha$ & $1.024( \pm 0.0 \%)$ & $1.044(-0.096 \%)$ & $1.065(-0.094 \%)$ & $1.051(-0.19 \%)$ & $1.063(-0.19 \%)$ \\
\hline
\end{tabular}

Table 2: Recalculated energy accommodation coefficient by using the complete heat transfer expression, Eq. (7), with real radius ratio and taking into account the dependence of $K_{F M}$ coefficient from the energy accommodation coefficient. The relative difference from the originally obtained accommodation coefficients, provided in Table 1, is given in the brackets.

\section{Accuracy Evaluation of Experimental Analysis}

In the experimental analysis, several approximations were made to extract the accommodation coefficient. However, they are not validated in detail because of a limitation of the experimental measurement in the simplified system. We will investigate here several approximations made during the experimental analysis to understand in detail the heat flux behavior between two concentric spherical shells.

\subsection{Free-molecular heat flux: effects of geometrical and physical parameters}

The complete analytical expression of the free-molecular heat flux for the case of the arbitrary shell's temperature ratio $\mathcal{T}$ and radius ratio $\mathcal{R}$ is given by Eq. (7).

If the radius of the external sphere is large compared to the internal one $(\mathcal{R} \rightarrow \infty)$, the case of the heat flux between a sphere and the surrounding gas, then the coefficient $K_{F M}$ in Eq. (7) tends to 1. In this case, Eq.(7) for $r=R_{H}$ gives the same results as Eq. (2) with $p=p_{0}$.

We evaluate now the value of coefficient $K_{F M}$ in Eq. (7) for the real radius ratio $\mathcal{R}=10$ and for several sets of measured temperature ratios and accommodation coefficients given in Table 1 . In the case of small temperature ratio $\mathcal{T}_{\min }=1.139$ with He, the deviation of $K_{F M}$ coefficient from unity is of the order of $0.01 \%$; while, in the case of large temperature ratio $\mathcal{T}_{\max }=1.543$ with $\mathrm{Xe}$, this deviation increases up to $0.15 \%$. Therefore, a posteriorly estimation of the coefficient $K_{F M}$ 's deviation from unity seems to be relatively small, so expression of Eq.(2) gives very accurate results for the used in the experimental setup radius ratio $\mathcal{R}=10$.

However, the $K_{F M}$ coefficient depends also on the accommodation coefficient, Eq. (7), and it might probably affect the fitting result. Therefore, the experimentally measured heat fluxes are re-analyzed by using the complete analytical expression, Eq. (7). From Table 2, it is clearly shown that neither the assumption of the infinitely large radius ratio $(\mathcal{R} \rightarrow \infty$ if $\mathcal{R}>10)$, nor the dependence of the $K_{F M}$ coefficient on $\alpha$ does not affect the results. Therefore, the implementation of Eq. (2) in the experimental analysis of the free-molecular flow regime is completely justified with maximum accuracy less than $0.2 \%$.

\subsection{Continuum heat flux: effects of geometrical and physical parameters}

Let now analyze the analytical expression of the heat transfer in the continuum flow regime. This expression takes part of the empirical fitting formula, 


\begin{tabular}{|ll|lllll|}
\hline \multicolumn{2}{|c|}{ cases } & 1 & 2 & 3 & 4 & 5 \\
\hline $\operatorname{He}$ & $\alpha$ & $0.280( \pm 0.0 \%)$ & $0.292( \pm 0.0 \%)$ & $0.307(-0.33 \%)$ & $0.321(-0.31 \%)$ & $0.337(-0.30 \%)$ \\
$\mathrm{Ar}$ & $\alpha$ & $0.842(-0.95 \%)$ & $0.855(-1.4 \%)$ & $0.842(-1.7 \%)$ & $0.844(-2.4 \%)$ & $0.862(-2.8 \%)$ \\
$\mathrm{Xe}$ & $\alpha$ & $1.021(-0.29 \%)$ & $1.031(-1.4 \%)$ & $1.041(-2.4 \%)$ & $1.021(-3.1 \%)$ & $1.024(-4.0 \%)$ \\
\hline
\end{tabular}

Table 3: Recalculated energy accommodation coefficient by using the empirical heat transfer expression with the continuum heat flux $q_{C}$, calculated using Eq. (12). The relative difference from the originally obtained accommodation coefficients, provided in Table 1 , is given in the brackets.

Eq. (3), which is used for the extraction of the accommodation coefficient in the case when the experimental pressure range is extended to higher pressure. Even though the heat transfers are measured at relatively low pressure condition around the near free-molecular regime, the heat transfer in the continuum limit could affect the results through Eq. (3). Initially, Eq. (4) is used with the constant thermal conductivity, calculated at temperature $T_{C}$ due to the large surface-area ratio of the external to the internal spherical shells $\mathcal{R}^{2}$ with the large radius ratio $\mathcal{R}[7,8]$. While, the complete analytical expression of the heat transfer in the continuum flow regime, Eq.(12), takes into account the dependence of the heat transfer coefficient on the temperature. It is clear that only coefficient $K_{C}$ makes the difference between two expressions.

In the limit of the small temperature difference between the spheres' surfaces $T_{H}-T_{C} \ll T_{C}$ or $\mathcal{T} \rightarrow 1, K_{C} \rightarrow 1$ and expression (12) for $r=R_{H}$ is equivalent to Eq.(4). In the cases of small temperature ratio $\mathcal{T}=1.139$, see Table 1 , the discrepancy coming from this $K_{C}$ factor becomes $4.5 \%, 5.6 \%, 5.9 \%$ for $\mathrm{He}$, $\mathrm{Ar}$, and Xe, respectively. However, for the largest temperature ratio between the surfaces and with Xe, it increases up to $22 \%$. Thus, the approximations of the heat transfer in the continuum limit might be a large error source in the experimental analysis.

The experimentally measured heat fluxes are re-analyzed by the analytical form, Eq. (12), instead of Eq. (4). The extracted accommodation coefficients are listed in Table 3. The discrepancy from the originally provided value, see Table 1, are also listed in (). It is clearly shown that the discrepancies are much larger for the conditions with large temperature differences since the temperature distribution between the shells affects the results. The difference in the gas species is resulting first from the difference in the thermal conductivity coefficient for different gases which is involved in the $q_{C}$ expression, Eq.(12). Then, for the same value of pressure, the rarefaction parameters $\delta_{0}$, Eq. (5), and so the flow regimes are different for the different gases: the lighter Helium is still in free molecular regime (smaller $\delta_{0}$ ), whilst the heavier Xenon is already in the transitional regime. Although there is a large difference in the evaluation of the heat transfer in the continuum limit between the analytical form and the original expression used in the experiment, the effect on the accommodation coefficient is less than $5 \%$. This should be because the heat transfers were measured in a low pressure environment, less than 1.6 Pa. However, it is recommended to use the analytical form $q_{C}\left(R_{H}\right)$ by Eq. (12), instead of Eq. (4), since it is more accurate and easy to integrate in the experimental analysis. 


\subsection{Revised expression for the heat flux interpolation}

To express the heat flux in the transitional regime, the empirical expression, Eq. (3), is useful and known to show quite good results $[8,7,9]$. In order to evaluate the accuracy of this empirical expression in detail, the numerical solution of the S-model kinetic equation is evaluated with the Hard Sphere (HS) model for a wide range of parameters; two radius ratios $\mathcal{R}=2$ and 10 , two temperature ratios $\mathcal{T}=1.1$ and 1.5 and three accommodation coefficients $\alpha=0.6,0.8$ and 1.0, i.e. 12 cases in total. Figure 1 shows the results of the numerical solution of the S-model kinetic equation by markers, and the empirical expression, Eq. (3), with $q_{F M}$ and $q_{C}$ calculated by Eq. (2) and Eq. (12), respectively, by dotted lines. It is to note that the dimensionless heat flux $q^{*}=q /\left(p_{0} v_{0}\right)$ is plotted. From Fig. 1, the empirical expression coincides with the numerical S-model results up to $\delta_{0} \sim 1$. However, they start to deviate by increasing the rarefaction parameter from $\delta_{0}=10^{-1}$ up to $10^{2}$, i.e. in the major part of the transitional regime up to the continuum flow regime. The heat flux from the empirical expression starts to decrease at smaller $\delta_{0}$ compared to the numerical results.

To make a better fitting function to reproduce the results of the S-model solutions, the following expression in a dimensionless form is proposed as a modification of the empirical expression, Eq. (3):

$$
\frac{1}{q^{*}}=\frac{1}{q_{F M}^{*}}+\frac{1}{\zeta q_{C}^{*}}, \quad \zeta=\frac{1}{1-\frac{c_{1}}{\delta_{0}+c_{2}}},
$$

where the dimensionless heat flux in the free-molecular $q_{F M}^{*}$ and continuum $q_{C}^{*}$ limits are expressed in Eqs. (37) and (23) in Ref. [9]. The factor $\zeta$ is introduced to improve the fitting quality in the transitional flow regime. The form of Eq. (13) is inspired by the expression of the dimensionless heat flux in the slip flow regime $q_{S}^{*}$, indicated as $q_{r}(r)$ in Eqs. (14) and (17) in Ref. [9]. In that paper, $q_{S}^{*}$ is obtained by integrating the energy balance equation with the temperature jump boundary conditions. This heat flux in the slip flow regime, $q_{S}^{*}$, was found to be different from $q_{C}^{*}$ by a factor of $\zeta_{s}=1 /\left(1+c / \delta_{0}\right)$, where $c$ is a constant defined by temperature jump coefficients, temperatures and radii of shells. However, this factor is not suitable as a correction term to the empirical expression, because the factor $\zeta_{s}$ vanishes in the free-molecular limit $\left(\delta_{0} \rightarrow 0\right)$, resulting in the finite limit value for $1 /\left(\zeta_{s} q_{C}^{*}\right)$, because $q_{C}^{*}$ is proportional to $1 / \delta_{0}$. Then, a constant is added to $\delta_{0}$ in $\zeta$ factor to avoid the problem, so now $1 /\left(\zeta q_{C}^{*}\right)$ vanishes in the free-molecular limit. The correction factor $\zeta$ goes to unity in the continuum limit $\left(\delta_{0} \rightarrow \infty\right)$.

Here, the coefficients $c_{1}$ and $c_{2}$ are analyzed to satisfy the dimensionless $\left(q^{*}\right)$ S-model results for 12 different cases, and it is suggested from fittings by the least-square method by the dimensionless form of heat flux that $c_{1}=1.04 \alpha \mathcal{T} / \mathcal{R}$ and $c_{2}=1.97 \alpha \mathcal{T} / \mathcal{R}$. The fitting results by this revised fitting function are plotted by solid lines in Fig. 1. It is clearly shown that the agreement between the S-model solutions and the revised fitting function are excellent for the whole range of the rarefaction parameter. It is also important to note that the revised 


\begin{tabular}{|c|lll|lll|lll|lll|}
\hline $\mathcal{R}$ & \multicolumn{9}{|c|}{2} & \multicolumn{5}{c|}{10} \\
\hline $\mathcal{T}$ & \multicolumn{3}{|c|}{1.1} & & \multicolumn{3}{c|}{1.5} & \multicolumn{3}{c|}{1.1} & & 1.5 \\
\hline$\alpha_{S}$ & 1.0 & 0.8 & 0.6 & 1.0 & 0.8 & 0.6 & 1.0 & 0.8 & 0.6 & 1.0 & 0.8 & 0.6 \\
\hline Empirical & 1.017 & 0.812 & 0.607 & 1.019 & 0.813 & 0.608 & 1.017 & 0.812 & 0.607 & 1.015 & 0.810 & 0.605 \\
Modified & 0.993 & 0.796 & 0.598 & 0.999 & 0.800 & 0.600 & 0.999 & 0.800 & 0.600 & 0.999 & 0.799 & 0.599 \\
\hline
\end{tabular}

Table 4: To check the effect of the heat flux expression for the transitional regime on the accommodation coefficient extraction procedure, the accommodation coefficients are extracted from the heat flux of the S-model solutions by using the empirical expression, Eq. (3), and the revised fitting function, Eq. (13).

fitting function reproduces very well the numerical solution for wide range of parameters; $1.1 \leq \mathcal{T} \leq 1.5$ and $2 \leq \mathcal{R} \leq 10$.

To validate the fitting procedure to obtain the accommodation coefficient by the revised function, the accommodation coefficient is extracted from the S-model numerical solutions by the same procedure as that used for the experimental data. The extracted value of the accommodation coefficient should be the same as that used initially in the numerical simulation, $\alpha_{S}$, if the extraction procedure is accurate enough. The revised function, Eq. (13), and the original empirical expression, Eq. (3), are employed as a fitting function and the extracted accommodation coefficients are compared. The data are fitted by the least-square method in the dimensionless forms; $q^{*}$ vs. $\delta_{0}$. The extracted accommodation coefficients are listed in Table 4. The accommodation coefficients, used in the numerical simulations, are denoted as $\alpha_{S}$ in the table. As expected from the discrepancies between the S-model solutions and the empirical expression in Fig. 1, extracted accommodation coefficients by the empirical expression can not reproduce the value originally used in the numerical simulation, $\alpha_{S}$. Compared to the empirical expression, the revised function reproduces the accommodation coefficients quite well. Even in the worst case where both $\mathcal{R}$ and $\mathcal{T}$ are small, the deviation is less than $1 \%$. Therefore, it is not highly accurate to use the empirical expression, Eq. (3), for the accommodation coefficient extraction procedure in the transitional regime.

The revised fitting function, Eq. (13), is employed to extract the accommodation coefficients from the experimental results. In the accommodation coefficient extraction procedure, the experimentally measured heat flux is fitted in the dimensional form. This is essential to avoid the effect from the measurement error in a low pressure condition [8]. In the dimensional form of Eq. (13), the dimensional heat fluxes $q_{F M}$ and $q_{C}$ instead of the dimensionless $q_{F M}^{*}$ and $q_{C}^{*}$ are expressed by using the dimensional form derived from Eq. (7) at the free-molecular flow limit and Eq. (12) at the continuum limit. The rarefaction parameter $\delta=\left(p R_{0}\right) /\left(\mu_{0} v_{0}\right)$ using the experimentally measured pressure $p$ is employed instead of $\delta_{0}$. Re-calculated accommodation coefficients by the revised fitting function are listed in Table 5. Compared with the results in Table 3, it is clearly shown that the function to express the heat flux in the transitional regime is as important as the consideration of the temperature distribution in the continuum limit in Section 4.2. 

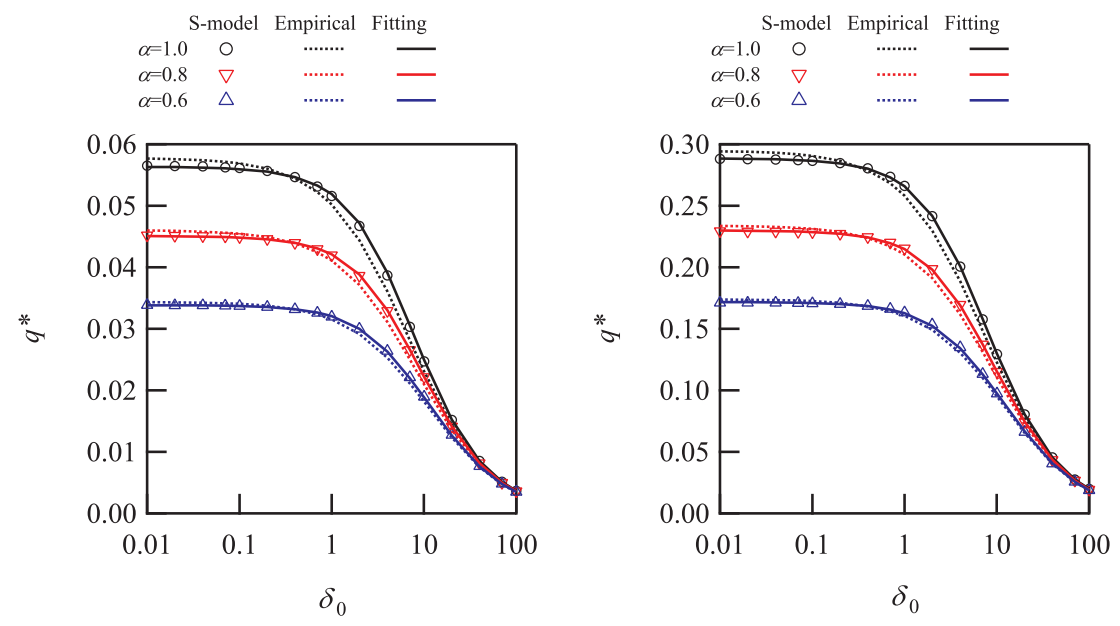

(a) $\mathcal{T}=1.1, \mathcal{R}=2$

(b) $\mathcal{T}=1.5, \mathcal{R}=2$
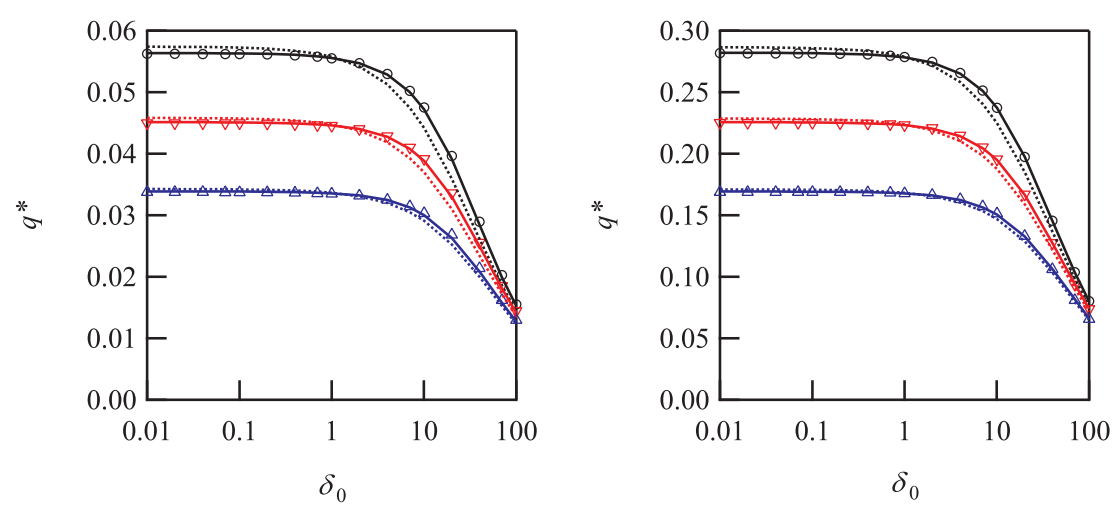

(c) $\mathcal{T}=1.1, \mathcal{R}=10$

(d) $\mathcal{T}=1.5, \mathcal{R}=10$

Figure 1: The dimensionless heat fluxes $q^{*}$ are plotted as a function of the rarefaction parameter $\delta_{0}$. The S-model solutions, shown by markers in all flow regimes, are compared with the empirical expression, represented by dotted lines, and the revised fitting function, shown by solid lines. For the accommodation coefficients $\alpha=1.0$, the results are plotted in black (S-model: $\bigcirc$ ), for $\alpha=0.8$, they are plotted in red (S-model: $\nabla$ ) and for $\alpha=0.6$, they are plotted in blue (S-model: $\triangle$ ), respectively.

\begin{tabular}{|ll|lllll|}
\hline \multicolumn{2}{|c|}{ cases } & 1 & 2 & 3 & 4 & 5 \\
\hline $\mathrm{He}$ & $\alpha$ & $0.278(-0.72 \%)$ & $0.290(-0.69 \%)$ & $0.305(-0.98 \%)$ & $0.319(-0.94 \%)$ & $0.335(-0.90 \%)$ \\
$\mathrm{Ar}$ & $\alpha$ & $0.804(-5.7 \%)$ & $0.816(-6.3 \%)$ & $0.809(-5.8 \%)$ & $0.810(-6.7 \%)$ & $0.828(-7.0 \%)$ \\
$\mathrm{Xe}$ & $\alpha$ & $0.938(-9.2 \%)$ & $0.952(-9.8 \%)$ & $0.963(-11 \%)$ & $0.951(-11 \%)$ & $0.958(-11 \%)$ \\
\hline
\end{tabular}

Table 5: Re-extracted energy accommodation coefficient by using the revised fitting function, Eq. (13), by using the more accurate expressions of the heat transfer in the free-molecular flow regime, Eq. (7), and in continuum flow regime, Eq. (12). The relative difference from the originally obtained accommodation coefficients, provided in Table 1, is given in the brackets. 


\subsection{Comparison between measured and simulated heat flux}

To validate the expressions of the heat flux between the concentric spherical shells, the energy accommodation coefficients are re-extracted from the experimental data, and the heat fluxes are numerically evaluated using the re-extracted results. The comparison between the measured heat flux as a function of pressure and the numerical results for three gases of $\mathrm{He}, \mathrm{Ar}$ and $\mathrm{Xe}$ for five cases of the surface temperatures are shown in Fig. 2. The numerical simulations are carried out using the S-model kinetic equation. Two different sets of the accommodation coefficient are employed; the original values extracted from the measurements listed in Table 1 and the corrected values, listed in Table 5, extracted by using the improvements, explained in the previous sections.

In Fig. 2 (a), (c) and (e), the dimensionless curves of the measured and simulated heat flux, $q^{*}=q /\left(p_{0} v_{0}\right)$, as the function of the pressure via the rarefaction parameter $\delta_{0}$ are presented. As it is clear from (a), the measured pressure range is restricted to the near free-molecular flow regime, where the dimensionless heat flux has the constant value, which depends only on the accommodation coefficient. However, in (c) and (e), the curves of the heat flux start to decreases, that means the flow is in the transitional, or even in the slip flow regime. From these figures, it is clear that the experimental data scattered around the lines, especially in the near free-molecular flow regime, due to the measurement difficulty as it was already explained in Section 4.3. The experimental data seemed to converge in the region where the lines curved. The comparison between the solid and dotted lines indicates that the corrected accommodation gives better agreement with the experimental results.

In Figs. 2 (b), (d) and (f), the dimensional quantities are plotted. This form is used for extracting the accommodation coefficient by the least square method. It is clearly shown that the agreement is much better from the original to corrected accommodation coefficient for the whole pressure range. Therefore, it is confirmed that the revised procedure suits well for the extraction of the accommodation coefficient. It is also important to note that even though the flat plate-shaped heater is assumed as a sphere in the experiment, as it was explained in Section 2, the experimental heat flux is well simulated by the numerical solutions of the S-model kinetic equation, indicating the validity of the assumption.

The corrected accommodation coefficients are also compared with those in the literatures $[3,15,16]$ for validation in Fig. 2. Considering the difference in surface conditions which is not known, the corrected values are in good agreement.

\section{Conclusion}

The measurement technique, called the low-pressure method, was analyzed by comparing the expressions, usually used by this technique, with the analytical expressions and numerical solution of the S-model kinetic equation. In the free-molecular flow limit, it is confirmed that the original expression coincides with the analytical form, and it is reasonable to assume the radius ratio 


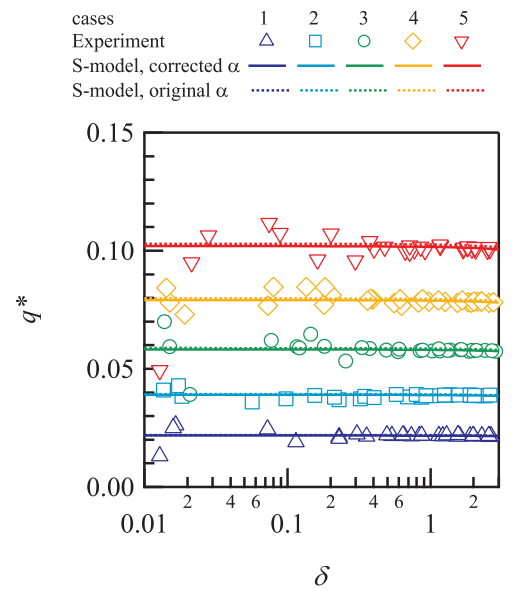

(a) He, dimensionless

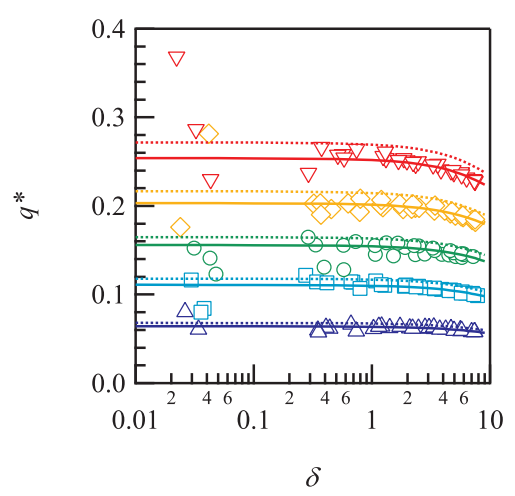

(c) Ar, dimensionless

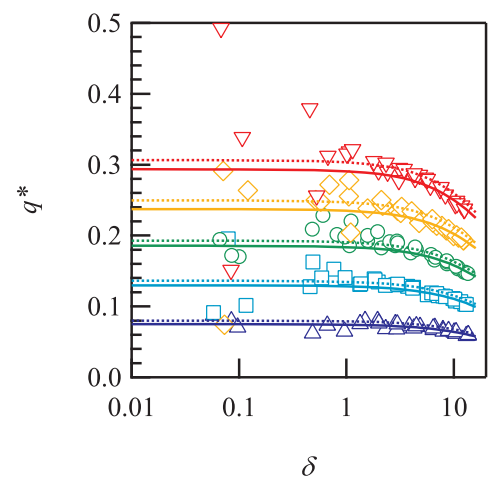

(e) Xe, dimensionless

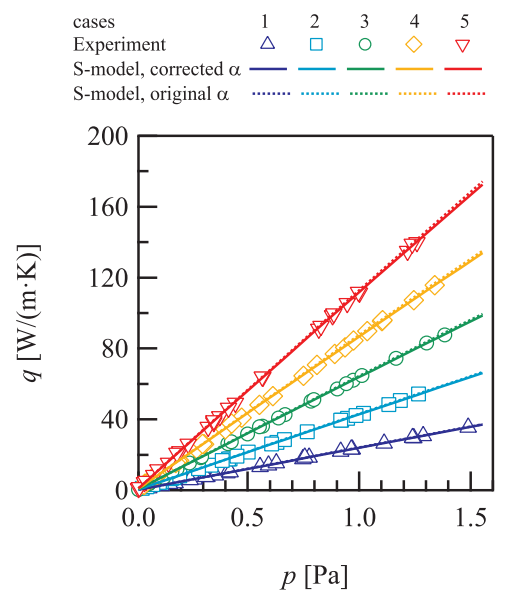

(b) He, dimensional

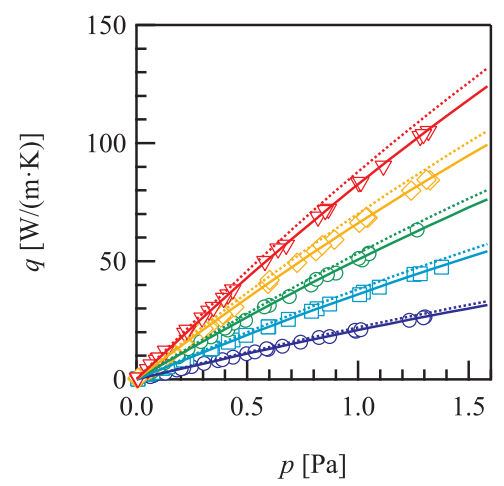

(d) Ar, dimensional

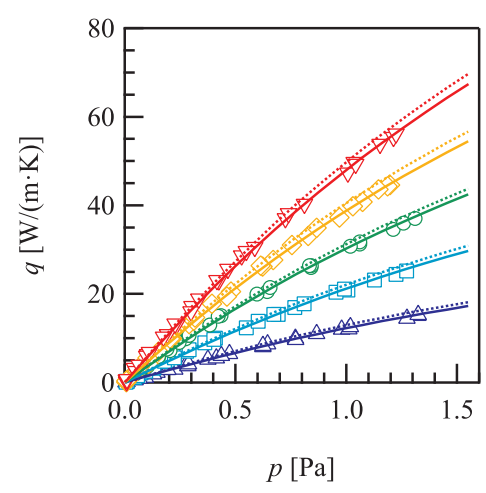

(f) Xe, dimensional

Figure 2: The heat flux as a function of pressure: comparisons between the experimental and numerical data with the dimensionless and the dimensional forms for the five cases. The numerical results by using the originally obtained accommodation coefficients, listed in Table 1 , and by using the corrected values, listed in Table 5 , are compared. 


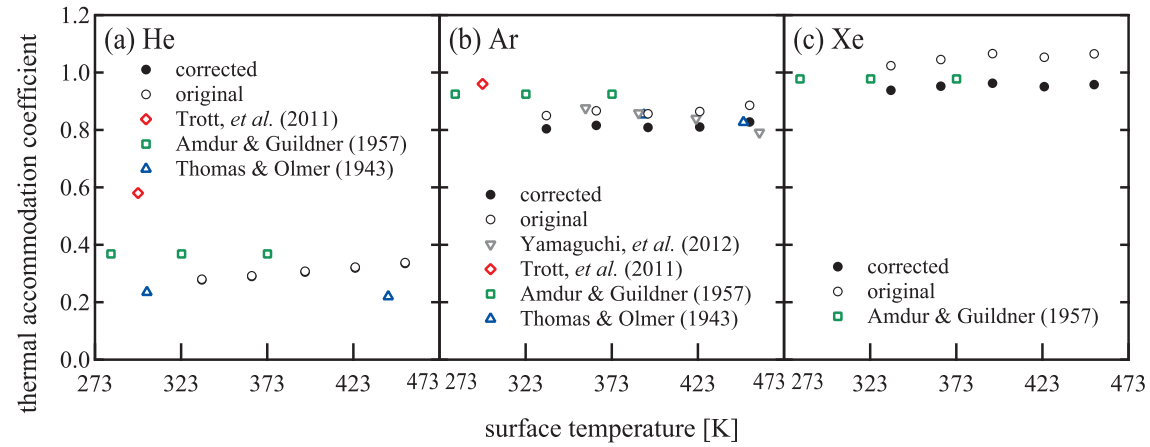

Figure 3: The corrected energy accommodation coefficients on the platinum surface are plotted with the original value for three gas species; (a) Helium, (b) Argon and (c) Xenon, and compared with data in the literatures.

to be infinite when it is about 10. In the continuum limit, it is important to consider the temperature dependence of the thermal conductivity coefficient. In the transitional regime, there is a small discrepancy between the empirical relation and the S-model kinetic solution. An empirical revised fitting function is proposed, which gives better fitting to the numerical results by the S-model kinetic equation. By employing this improved expression, the calculated accommodation coefficient changes about $12 \%$ in maximum. The improvement have larger effect in the case of the larger temperature ratio and the larger rarefaction parameter regime. The use of the corrected accommodation coefficient allows to reproduce well the measured heat flux.

\section{Acknowledgments}

This work was partially supported by JSPS KAKAENHI Grant Number 16K14157. This work was also granted access to the HPC resources of AixMarseille Université financed by the project Equip@Meso (ANR-10-EQPX-2901) of the program "Investissements d'Avenir" supervised by the Agence Nationale pour la Recherche. The authors would like to thank Mr. Takamasa Imai, Mr. Tadashi Iwai and Mr. Akira Kondo for their support on the measurements.

[1] F O Goodman and H Y Wachman. Dynamics of Gas-Surface scattering. Academic Press, New York, NY, USA, 1976.

[2] S. C. Saxena and R. K. Joshi. Thermal Accommodation and Adsorption Coefficients of Gases. Hemisphere, New York, 1981.

[3] W. M. Trott, J. N. Castañeda, J. R. Torczynski, M. A. Gallis, and D. J. Rader. An experimental assembly for precise measurement of thermal accommodation coefficients. Rev. Sci. Instrum., 82:0355120, 2011. 
[4] M Grau, F Völkein, A Meier, C Kunz, J Heidler, and P Woias. Method for measuring thermal accommodation coefficients of gases on thin film surfaces using a mems sensor structure. J. Vac. Sci. Technol. A, 34(4):041601, 2016.

[5] P Spijker, A J Markvoort, S V Nedea, and P A J Hilbers. Computation of accommodation coefficients and the use of velocity correlation profiles in molecular dynamics simulations. Phys. Rev. E, 81:011203, Jan 2010.

[6] Z Liang and P Keblinski. Parametric studies of the thermal and momentum accommodation of monoatomic and diatomic gases on solid surfaces. International Journal of Heat and Mass Transfer, 78:161 - 169, 2014.

[7] H Yamaguchi, T Imai, T Iwai, A Kondo, Y Matsuda, and T Niimi. Measurement of thermal accommodation coefficients using a simplified system in a concentric sphere shells configuration. Journal of Vac. Sci. Technol. A, 32(6):061602, 2014.

[8] H Yamaguchi, K Kanazawa, Y Matsuda, T Niimi, A Polikarpov, and I Graur. Investigation on heat transfer between two coaxial cylinders for measurement of thermal accommodation coefficient. Physics of Fluids, 24(062002), 212.

[9] M T Ho and I Graur. Heat transfer through rarefied gas confined between two concentric spheres. International Journal Heat and Mass Transfer, 90:58-71, 2015.

[10] E M Shakhov. Generalization of the Krook kinetic relaxation equation. Fluid Dyn., 3(5):95-96, 1968.

[11] G. S. Springer. Heat transfer in rarefied gases. In T. F. Irvine and J. P. Harnett, editors, Advanced in heat transfer, pages 163-218. Academic, New York, 1971.

[12] F. S. Sherman. A survey of experimental results and methods for the transitional regime of rarefied gas dynamics. In J. A. Lauermann, editor, Rarefied Gas Dynamic, Proc. 3rd Int. Symp. on Rarefied Gas Dynamics, volume II, pages 228-260, New York, 1963. Academic Press.

[13] C Cercignani. Mathematical methods in kinetic theory. Premuim Press, New York, London, 1990.

[14] G A Bird. Molecular Gas Dynamics and the Direct Simulation of Gas Flows. Oxford Science Publications, Oxford University Press Inc., New York, 1994.

[15] I Amdur and L A Guildner. Thermal accommodation coefficients on gascovered tungsten, nickel and platinum. Journal of the Americal Chemical Society, 79(2):311-315, 1957. 
[16] L. B. Thomas and F. Olmer. The accommodation coefficients of he, ne, a, $\mathrm{h} 2, \mathrm{~d} 2$, o2, co2, and hg on platinum as a function of temperature. J. Am. Chem. Soc., 65(6):1036-1043, 1943. 